\title{
EXCITONIC INSTABILITIES OF DEFORMABLE LATTICE - FROM SELF-TRAPPING TO PHASE TRANSITION
}

\author{
Y. TOYOZAWA \\ Department of Physics, Chuo University \\ Kasuga 1-13-27, Bunkyo-ku, Tokyo 112, Japan
}

\begin{abstract}
The optically created exciton will be self-trapped if its coupling to phonons is strong enough, and will moreover be self-decomposed if the electron and the hole couple to phonons in an opposite way. The bistability between the parity-conserved and parity-broken self-trapped excitons was observed in alkali halides. The situation is most dramatic if the bistability between the parity-broken self-trapped exciton and the ground state (with no exciton) comes into play since the electron-hole pairs may then be spontaneously generated at every lattice site, resulting in the electronic and structural phase transition. The neutral to ionic phase transition observed in a few organic charge transfer compounds under applied pressure or decreasing temperature can be considered as an example. Recent experiment revealed that TTF-chloranil, among others, is subject to photo-induced transient phase change over hundreds of unit cells per one photon. The dynamics of this process can be described in terms of self-trapping and self-multiplication of a photo-generated charge transfer exciton along the chain through the attractive dipolar interaction. This description of phase transition in terms of exciton dynamics will provide a new paradigm of materiology.
\end{abstract}

PACS numbers: $64.60 .-\mathrm{i}, 71.35 .+\mathrm{z}, 71.38 .+\mathrm{i}$

\section{Introduction}

Aggregation of atoms to form molecules and solids originates in the interatomic forces mediated by outermost electrons being extended or transferred to nearby atoms. Thus the atomic configuration on one hand and the electronic state on the other hand depend upon each other. This correlation is concealed in the ground state of a molecule or insulator where everything is balanced self-consistently, except for small vibration of atoms around their equilibrium positions which is immediately followed by a small fluctuation of electronic charge. However, it is revealed once an electron is brought into an excited state by radiation or particles; the sudden change in charge distribution or chemical bond will destroy the balance 
of interatomic forces and cause atomic rearrangement - lattice relaxation in the case of solids.

However, this reasoning which is obvious in molecules of finite size does not straightforwardly apply to solids of infinite size since the photo-excited pair of an electron and a hole, or an exciton, is usually considered to be in an extended state in conformity with the translational symmetry of the lattice and does not contribute a finite change in charge distribution. In fact, the Bloch state has been considered to be the best starting point to study the electronic properties of solids, in particular, of normal metals and semiconductors.

If for some reason the excited entity - electron, hole or exciton - becomes localized, it gives finite change in charge distribution which in turn causes finite local lattice distortion. The latter may be large enough to stabilize the electronic localization we assumed. Such a state is called self-trapped state which will be our next subject.

\section{Self-trapped state}

More elaborate argument is needed to conceptually distinguish the self-trapped state from the delocalized state. First of all, it is meaningless to distinguish between them in case of a molecule; as mentioned before, finite rearrangement of atoms always takes place after the electronic excitation since the electronic charge and hence its change is localized. In solids, one has to study the adiabatic potential of the lattice for the excited electronic state; if there is a potential barrier between the delocalized electronic state with no (or small, at most) lattice distortion and the localized electronic state with significant lattice distortion, one can distinguish the two states (the bistability - one being stable and the other metastable) within the adiabatic approximation - more exactly speaking, when the barrier is so high that the quantum-mechanical tunneling of lattice coordinates between the two states takes time long compared to the time scale of our concern: the time for the electronic transition and the time for the lattice relaxation, etc.

A simple but perspective way of studying the adiabatic potential is the continuum model [1], in which one replaces the distortion of the discrete lattice by (i) elastic continuum with isotropic dilation field $\Delta(r)$ to describe the electron-acoustic phonon coupling through the deformation potential constant $\xi$ and (ii) dielectric continuum with electrostatic potential $\Phi(r)$ due to displacement polarization to describe the electron-optical phonon coupling. For a single electron (hole) in the conduction (valence) band interacting with the acoustic and optical phonon fields, one can write the adiabatic energy in the functional form:

$$
\begin{gathered}
W[\psi(r), \Delta(r), \Phi(r)]=\int \mathrm{d} r \psi(r)\left(-\hbar^{2} / 2 m\right) \nabla^{2} \psi(r) \\
+\int \mathrm{d} r \psi(r)^{2} \xi \Delta(r)+\int \mathrm{d} r \psi(r)^{2} e \Phi(r) \\
+\int \mathrm{d} r(C / 2) \Delta(r)^{2}+\int \mathrm{d} r(\varepsilon / 8 \pi)(\nabla \Phi(r))^{2} .
\end{gathered}
$$

Here $e$ is the electronic charge, $m$ - the band effective mass, $C-$ an elastic constant and $\varepsilon^{-1} \equiv \varepsilon_{\infty}^{-1}-\varepsilon_{0}^{-1}$ with $\varepsilon_{0}$ and $\varepsilon_{\infty}$ denoting static and high frequency 
dielectric constants, respectively. Minimization of Eq. (1) with respect to the envelope wave function $\psi(r)$ gives the lowest eigenstate of the Schrödinger equation as a functional of $\Delta(r)$ and $\Phi(r)$, and putting this $\psi$ into (1) gives the adiabatic potential $W[\Delta, \Phi] \equiv W[\psi[\Delta, \Phi], \Delta, \Phi]$ in the infinite dimensional configuration coordinate space $[\Delta(r), \Phi(r)]$. The first step is difficult to perform explicitly. However, if one is interested only in the extrema of the adiabatic potential, one can invert the order of extremizations. Extremizing first with respect to $\Delta(r)$ and $\Phi(r)$ for fixed $\psi(r)$, one obtains the explicit solution: $\Delta(r)=-(\xi / C)|\psi(r)|^{2}$ and $\nabla^{2} \Phi(r)=(4 \pi e / \varepsilon)|\psi(r)|^{2}$. Putting this into Eq. (1), one obtains $W$ as a functional of $\psi$ :

$$
\begin{aligned}
& W[\psi]=\int \mathrm{d} r \psi(r)\left(-\hbar^{2} / 2 m\right) \nabla^{2} \psi(r)-(1 / 2) \int \mathrm{d} r \int \mathrm{d} r^{\prime} \psi(r)^{2} \\
& \quad \times\left[\left(\xi^{2} / C\right) \delta\left(r-r^{\prime}\right)+\left(e^{2} / \varepsilon\right)\left|r-r^{\prime}\right|^{-1}\right] \psi\left(r^{\prime}\right)^{2} .
\end{aligned}
$$

It is to be noted that the integral kernel [] of the self-interaction - the effect of the electron-induced lattice distortion acting upon the electron itself - consists of short and long range parts originating in acoustic and optical phonon fields, respectively. This difference in the force range and the dimensionality of the lattice play important roles for the above mentioned bistability as we shall see.

The extrema of the adiabatic potential are obtained by extremizing (2) with respect to $\psi$. Putting into (2) the trial wave function: $\psi(r)=(a / \sqrt{2})^{-3 / 2}$ $\mathrm{x} \exp \left[-\pi(r / a)^{2}\right]$ in which the orbital radius $a$, the only variational parameter, is to be varied between $a_{0}$ (complete localization; note that $\psi$ represents the envelope wave function) and $\infty$ (complete delocalization), one obtains $W$ as a function of $a$ :

$$
W[\psi]=W(a)=B\left(a_{0} / a\right)^{2}-E^{(\mathrm{ac})}\left(a_{0} / a\right)^{3}-E^{(\mathrm{op})}\left(a_{0} / a\right),
$$
where $B \equiv 3 \pi \hbar^{2} / 2 m a_{0}^{2}, E^{(\mathrm{ac})} \equiv \xi^{2} / 2 C a_{0}^{3}$ and $E^{(\mathrm{op})} \equiv e^{2} / \varepsilon a_{0}$ denote the electron kinetic energy and the lattice relaxation energies due to the acoustic and optical phonon fields, each for complete localization. Defining $g_{\mathrm{s}} \equiv E^{(\mathrm{ac})} / B$ and $g_{1} \equiv E^{(\mathrm{op})} / B$, the short and long range electron-phonon coupling constants, one finds the "phase diagram" describing the number and the natures of extrema of Eq. (3) within the interval: $0 \leq \lambda \equiv a_{0} / a \leq 1$, as shown in Fig. 1. With $g_{1} \ll 1$ as is mostly the case except in narrow band materials, the stable state of the electron changes from the delocalized $(\lambda \ll 1)$ or nearly free state, $F$, accompanied mainly by polarization (the so-called large (radius) polaron) - to the completely localized $(\lambda=1)$ or self-trapped state, $S$, accompanied by large lattice distortion of the both types (small (radius) polaron), abruptly across the discontinuity line shown by a solid line. On both sides of this line are the bistable regions $F(S)$ and $S(F)$ where the stable and the metastable (shown in parentheses) states are separated by a potential barrier (the third extremum representing the saddle point of the adiabatic potential). That the bistability is triggered by the short range interaction is obvious from Eq. (3) as well as from the termination of the discontinuity line in Fig. 1 without reaching the $g_{1}$ axis. In the remaining regions, there is the only stable state, $F$ or $S$.

The above prediction is consistent with experimental studies on the behaviors of electrons, holes and excitons in a variety of insulators, as assigned to each region [2]. First of all, these carriers can be rather distinctly classified into mobile $F$ 


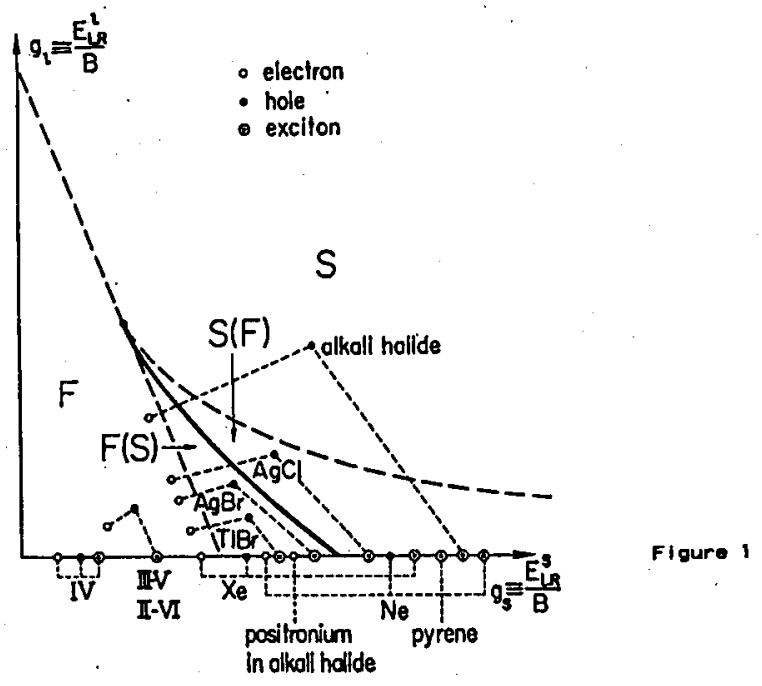

Fig. 1. Predicted phase diagram for an electron in the phonon fields, with assignment of electrons, holes and excitons in typical solids according to their behaviors.

type and immobile $S$ type, in view of their transport properties and optical behaviors. More direct confirmation of the discontinuity line has been provided by the abrupt change from $F$ to $S$ type under the continuous change in applied pressure or composition of mixture. In the region of bistability, the measurement has been done on the energy difference between the stable and metastable states through their temperature dependent populations and on the barrier height through the temperature dependence of the activated process.

In Fig. 1, the exciton has been plotted always on the abscissa because of its neutrality ( $e=0$ and $g_{1}=0$ ). The absence of the $S$ region on the abscissa means that the $F$ state of an exciton is either stable or metastable but never unstable. The situation differs completely in one-dimensional lattice, where the exponent 3 on the second term of Eq. (3) is to be replaced by 1 as is obvious from Eq. (2). The short range coupling in 1-dimensional lattice plays the same role as the long range coupling does in 3-dimensional lattice. There is the only minimum in the adiabatic potential, which changes continuously from the $F$-like to $S$-like state as the coupling constant $g_{1}$ increases.

\section{Self-decomposition of an exciton}

If one takes into account the internal structure of the exciton so far ignored, one finds the new features in the exciton instability in the deformable lattice. The electron and the hole interact with the optical phonon field in opposite way because of their opposite charge. They will induce polarization fields of opposite direction around them, forming the polaron pairs. The result is to increase the screening constant for the electron-hole Coulomb attraction from $\varepsilon_{\infty}$ to $\varepsilon_{0}$ (at distance greater than the polaron radii) and hence to decrease the binding energy. 
On the other hand, the deformation potential constants for the electron and the hole, $\xi_{\mathrm{e}}$ and $\xi_{\mathrm{h}}$, are independent parameters. If they have opposite signs, they will induce lattice dilations of opposite sign around them, resulting in the short range repulsive force between them. If $|\xi|$ 's are large enough, it is possible to have a pair of electron and hole self-trapped at a distance where this phonon mediated short range repulsion counterbalances the long range Coulomb attraction. This partially self-decomposed self-trapped exciton (STE) - the parity-broken STE, which was initially predicted [3] as a theoretical possibility, finds now the experimental counterpart: recent experimental and theoretical studies [4] revealed that STE in majority of alkali halides are in the off-center configuration - type III STE according to Kan'no et al.; there are also the on-center STE's - type I - in other alkali halides.

\section{Instability across the band gap - phase transition}

We have so far confined ourselves to the electronic states of a pair excitation. However, the total stabilization energy, $E_{\text {st }}\left(=E^{(\mathrm{ac})}-B\right.$ according to the model of Sec. 2, see Eq. (3)), of an exciton due to the lattice relaxation throughout the self-trapping and (if any) the self-decomposition processes, can in principle be as large as comparable to the optical energy $E_{0}$ needed to create the exciton in the $F$ state, since they are independent parameters of the material. In fact, the anomalously small quantum yield of luminescence from STE in some insulators indicates the importance of the nonradiative multiphonon annihilation of STE which takes place around the crossing points of the adiabatic potentials for the ground and the excited (with one exciton) states of the insulator [5].

When $E_{\text {st }}$ were to exceed $E_{0}$, namely, when $E_{\mathrm{R}} \equiv E_{0}-E_{\text {st }}$, the energy of the relaxed excited state (STE in its lowest state) referred to the ground state of the crystal as zero, were to become negative, the STE would be spontaneously generated at every lattice site, which amounts to the electronic and structural phase transition of the entire crystal. If the STE were of the parity broken type, the new ground state with electrons and holes on alternate sites would be the charge transferred state as compared with the ground state we started from.

Of course one must be more elaborate in making speculation on such a dramatic change [6]. Let us first consider a pair of STE's situated at the $i$-th and $j$-th sites. They have the interaction energy $v_{i j}$ consisting of direct electronic and indirect phonon-mediated interactions. Let us then proceed to the cluster of $n$ STE's. Assuming for simplicity that the interaction energy among these $n$ STE's is the sum of the pair interactions, one can write down the energy of the cluster as

$$
E_{\mathrm{R}[n]}=n E_{\mathrm{R}}+V_{[n]}, \quad V_{[n]} \equiv \sum_{i<j} v_{i j}
$$

where the summation over $i$ and $j$ is to be extended over the sites occupied by STE. The shape of an energetically favorable cluster for a given size $n$ should be that which makes best use of the attractive interactions $\left(v_{i j}<0\right)$ to maximize the binding energy $-V_{[n]}$. If the inequality

$$
E_{0}>E_{\mathrm{R}[n]}=n E_{\mathrm{R}}+V_{[n]}
$$


holds, it is energetically possible for an optical exciton to relax in the deformable lattice and proliferate to form such a cluster.

If the inequality (5) holds even in the limit of $n \rightarrow \infty$ with the cluster assumed to cover the entire lattice, namely, if the inequality

$$
\begin{aligned}
& \lim _{n \rightarrow \infty} E_{0} / n=0>E_{\mathrm{R}}-W_{1}, \\
& W_{1} \equiv \lim _{n \rightarrow \infty}\left(-V_{n} / n\right)=-\sum_{j \neq 0} v_{0 j} / 2
\end{aligned}
$$

holds, it not only is possible but does occur that the STE is spontaneously generated on every site, even without the optical excitation (note that $E_{0}$ plays no role in the inequality (6)). In Eq. (7), we assumed the convergence of the limiting procedure - a finite value of the cohesive energy per one STE, and made use of the translational symmetry of the lattice on the right hand side. Equation (6) means that the phase transition by excitonic instability takes place when the STE energy $E_{\mathrm{R}}$ becomes smaller than its cohesive energy $W_{1}$, instead of becoming negative as stated in the beginning of this section. For the purpose of seeking for the phase transition by decreasing the energy gap in any way, this requirement with finite $W$ is far less oppressive than was imagined initially. A possibly easier approach to the same goal may be to look for those materials with greater attractive interaction between STE's.

Of course, the nature always prefers the state with the lowest (free) energy, and it might be nothing more than the academic interest to imagine an alternative higher energy state which has not been preferred. Nevertheless, it is at least instructive to imagine the alkali halide, typical ionic crystal, to be formed as the result of spontaneous generation of the charge transfer excitons, with electrons and holes at halogen and alkali sublattices, respectively, on the fictitious lattice of neutral alkali and halogen atoms. Less trivial, but less known example may be the Wolffram's red, a quasi-one-dimensional material of which the skeleton of a chain would, according to a simple-minded fictitious model, consist of alternate array $\left(\mathrm{Cl}^{-}-\mathrm{Pt}^{3+}-\mathrm{Cl}^{-}-\mathrm{Pt}^{3+}\right)^{n}$. However, the stable structure realized in nature is $\left(\mathrm{Cl}^{-}-\mathrm{Pt}^{4+}-\mathrm{Cl}^{-}-\mathrm{Pt}^{2+-}\right)^{n}$, which is obtained from the former by spontaneous generation of holes and electrons at alternate $\mathrm{Pt}$ sites with corresponding contraction and elongation of the distance to $\mathrm{Cl}^{-}$. It can be described as Peierls transition from the metallic state to an insulator with charge density wave with doubled lattice period [7].

\section{Dynamics of self-multiplication of exciton and the photo-induced phase transition}

We will now describe the neutral-to-ionic (NI) phase transition in TTF-chloranil, a quasi-one-dimensional charge transfer compound, in the context of the preceding argument. The point-charge model proposed by Torrance et al. [8] gives a very nice and systematic explanation on the energy of a charge transfer exciton as well as on the NI transition observed in a series of similar compounds. With $I$ and $A$ denoting the ionization of a donor molecule $(D)$ and the electron affinity of an acceptor molecule $(A)$, respectively, and with $\alpha$ for 
the Madelung constant of a one-dimensional chain, and under neglection of intermolecular electron transfer energy, the energy to create a charge transfer exciton is given by $E_{0}^{N}=I-A-\left(e^{2} / a\right)$ and $E_{0}^{I}=A-I+(2 \alpha-1)\left(e^{2} / a\right)$ for $N$ phase $\left(D^{0} A^{0} D^{0} A^{0} \ldots\right)$ and $I$ phase $\left(D^{+} A^{-} D^{+} A^{-} \ldots\right)$, respectively. One readily finds, by a simple electrostatic argument, that the chain is in $N$ or $I$ phase according as $\Delta \equiv I-A-\alpha e^{2} / a \neq 0 . N \rightarrow I$ transition takes place since $a$ decreases as temperature is lowered or applied pressure increases. It is to be noted that $E_{0}^{N}=E_{0}^{I}$ at the transition point: $\Delta=0$. Plotting observed values of $E_{0}$ versus $\Delta$ for similar compounds fits very well with the theoretically predicted V-shape, with $N$ and $I$ phase compounds on the right and left sides, respectively, of the cusp at $\Delta=0$. TTF-chloranil, being in $N$ phase at room temperature (slightly on the right side), is brought into the $I$ phase below $T_{\mathrm{c}}=80 \mathrm{~K}$ above $p=11 \mathrm{kbar}$.

The first step beyond the point charge model is to take account of the electron transfer energy $t$ between the neighboring $D$ and $A$. This results in the hybrid bands formed from the highest occupied molecular orbital of $D$ and the lowest unoccupied molecular orbital of $A$. The coefficients of hybridization and the occupation of the bands are to be determined by minimizing the total energy within the unrestricted Hartree-Fock (UHF) approximation. The long range part of the Coulomb interaction among electrons and ions is fully taken into account as in the point charge model but in contradistinction to the Hubbard model since this part plays an essential role for the electrostatic instabilization of the lattice to be discussed later. Solving the integral equations for self-consistency, one obtains [9] two solutions: " $N$ " and " $I$ " within a small region on the $(\Delta, t)$-plane and the only solution in the outside region. The solution " $N$ ", in which the $D$-dominant band is filled by up and down spins and the $A$-dominant band is empty, represents the $N$ phase. In the solution " $\Gamma$ ", one obtains non-vanishing spin on the $D$ and $A$ sublattices: the lower of the $A$-dominant bands is filled by up spins and the lower of the $D$-dominant band is filled by down spins, or vice versa. It represents the ionic phase with antiferromagnetic order, the low lying excitation mode being the spin wave rather than the excitation to the upper bands. This ionic lattice of one-dimensionality is subject to Coulomb instability against the dimerization to form $\left(D^{+} A^{-} \cdot D^{+} A^{-} \cdot \ldots\right)$ (essentially the same as the spontaneous polarization in ferroelectrics), in agreement with observation. The discontinuity line on the $(\Delta, t)$-plane, across which the first order transition between the two phases takes place, extends almost upward from the point $(0,0)$ and terminates at $t /\left(e^{2} / a\right) \approx 0.2$. The metastable electronic state, coexistence with the stable state within the above-mentioned region on both sides of the discontinuity line, manifests itself in the photo-induced transient phase transition as will be seen in the following.

A photo-generated exciton in this one-dimensional chain will immediately be self-trapped because of the absence of potential barrier (Sec. 1), and will moreover lose its parity in such a charge transfer compound, even in the $N$ phase with inversion symmetry. A simple-minded picture for the relaxed exciton in the strong coupling case would be a $D^{+} A^{-}$dimer in the neutral matrix, or a $D^{0} A^{0}$ dimer in the ionic matrix, which in effect has a permanent dipole along the chain in either phase. This dipole will produce strong electric field 
on neighboring sites so as to induce similar dipoles with the same orientation. One-dimensionality is favorable for this induction to proceed: the dipole-dipole interaction: $v_{i j}=\mu^{2}\left(1-3 \cos ^{2} \theta\right) / R_{i j}^{3}$ is always negative because the dipole moment is oriented parallel to the chain $(\theta=0)$, thus promoting and stabilizing the multiplication of STE.

This is exactly the situation emphasized to be favorable in Sec. 4. Applying that general argument to the present case [6], one can write the dipolar interaction as $v_{0 j}=v_{01} / j^{3}, v_{01} \equiv-2 \mu^{2} / 2 a$. Since the binding energy of the cluster of $n$ connected STE's are given by $V_{n}=\sum_{j=1}^{n-1}(n-j) v_{0 j}$, the cohesive energy per one STE is given, according to Eq. (7), by

$$
W_{1}=-\sum_{j=1}^{\infty} v_{0 j}=\alpha_{3}\left|v_{01}\right|, \quad \alpha_{3}=\sum_{j=1}^{\infty} j^{-3}=1.202 .
$$

The value of $E_{0}$ for the intersection point of the boundary lines of inequalities (5) and (6) is given, after elimination of $E_{\mathrm{R}}$, by

$$
E_{0}^{n, \infty}=n W_{1}+V_{n} \equiv W_{n}=-\sum_{j=1}^{n-1} j v_{0 j}-\sum_{j=n}^{\infty} v_{0 j} .
$$

Putting this $W_{n}$ into (5), one obtains

$$
E_{\mathrm{R} n}=n(\gamma-1) W_{1}+W_{n}, \quad \gamma \equiv E_{\mathrm{R}} / W_{1} .
$$

$E_{\mathrm{R} n}$ as a function of $n$ tends asymptotically, as $n \rightarrow \infty$, to the straight line: $E_{\mathrm{R} n}=W_{\infty}+n(\gamma-1) W_{1}$, where

$$
W_{\infty}=-\sum_{j=1}^{\infty} j v_{0 j}=\alpha_{2}\left|v_{01}\right|, \quad \alpha_{2}=\sum_{j=1}^{\infty} j^{-2}=1.645 .
$$

Making use of Eqs. (8)-(11), one can plot $E_{\mathrm{R} n}$ as a function of $n$ for typical values of the stability parameter: $\gamma=1.1$ (stable phase), 1.0 (transition point) and 0.9 (unstable phase), as shown in Fig. 2. Let us denote by $n_{0}$ the greatest integer before

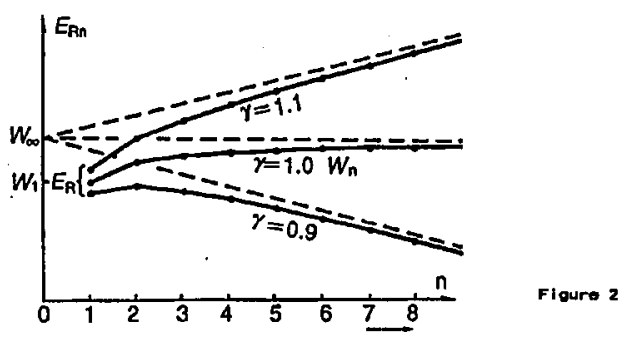

Fig. 2. The energy of a cluster of self-trapped excitons as a function of the cluster size.

the intersection of $E_{\mathrm{R} n}$ versus $n$ line for a stable phase $(\gamma>1)$ with the horizontal line: $E_{\mathrm{R} n}=E_{0}$. A free exciton of energy $E_{0}$ created optically will (under most favorable situation) be dynamically multiplied into the cluster of $n_{0}$ STE's within 
a short time. With $\gamma<1, E_{\mathbf{R} n}$ becomes negative for large $n$, implying that the ground state from which we started is unstable since STE's would be spontaneously generated even without the optical excitation. The situation is shown in Fig. 3 in the form of phase diagram on the $\left(E_{0}, E_{\mathrm{st}}\right)$-plane. The hatched region $U$ represents the unstable state. The stable region can be divided into sub-regions $M_{1}, M_{2}, \ldots$, $M_{n}, \ldots$ according to how many STE's can be generated (how many unit cells can be transiently brought into the metastable phase) per one optical exciton. One can transform, by one photon, as much region as one wants into the metastable phase as one approaches the transition point: $\gamma \rightarrow 1+0$, in conformity with the observation [10] of hundreds of unit cells transformed into the metastable $N$ state per one photon absorbed by the stable $I$ state just below $T_{\mathrm{c}}$.

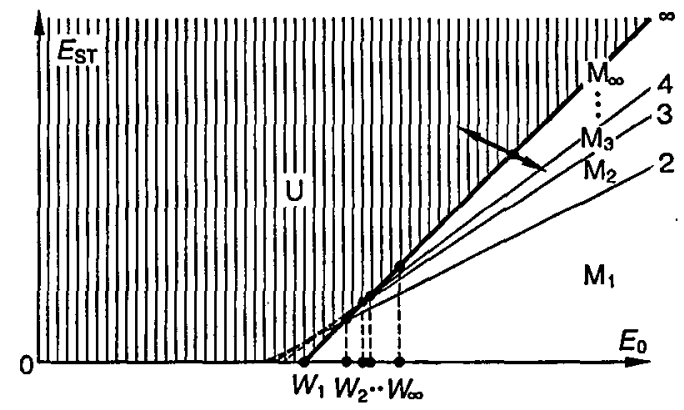

Fig. 3. Phase diagram for self-multiplication of an optically produced exciton.

Figure 3 can be applied both to $N$-phase or $I$-phase, whichever may be stable. How can one then explain the experimental fact [10] that the metastable $I$ state formed by photo-excitation of the $N$ state is undetectably short-lived, in contrast to the above mentioned case in which the metastable $N$ state is as long-lived as milliseconds? The answer is given by thermodynamic argument. Let us imagine the free energy versus temperature curves for the $N$ - and $I$-states, which should cross each other at $T_{\mathrm{c}}$. Photo-excitation of the $I$-phase just below $T_{\mathrm{c}}$ will generate a region of $N$ phase, which however may have been locally heated above $T_{\mathrm{c}}$ during the relaxation-multiplication process and hence be in the stable state until it is cooled again below $T_{\mathrm{c}}$ by slow outflow of heat. In contrast, the transient $I$ phase region generated by photo-excitation of the $N$ phase is even more above $T_{\mathrm{c}}$, being in the metastable electronic state which is very short-lived.

\section{References}

[1] Y. Toyozawa, in: Relaxation of Elementary Excitations, Eds. R. Kubo, E. Hanamura, Springer, Berlin 1979, p. 3.

[2] M. Ueta, H. Kanzaki, K. Kobayashi, Y. Toyozawa, E. Hanamura, Excitonic Processes in Solids, Springer, Berlin 1986, Ch. 4.

[3] A. Sumi, J. Phys. Soc. Jpn. 43, 1286 (1977). 
[4] K.S. Song, R.T. Williams, Self-Trapped Excitons, Springer, Berlin 1993; Y. Kayanuma, in: Defect Processes Induced by Electronic Excitation in Insulators, Ed. N. Itoh, World Scientific, Singapore 1989, p. 13; T. Matsumoto, T. Kawata, A. Miyamoto, K. Kan'no, J. Phys. Soc. Jpn. 61, 4229 (1992).

[5] Y. Toyozawa, J. Phys. Soc. Jpn. 58, 2646 (1989).

[6] Y. Toyozawa, Solid State Commun. 84, 255 (1992).

[7] H. Tanino, K. Kobayashi, J. Phys. Soc. Jpn. 52, 1446 (1983).

[8] J.B. Torrance, J.E. Vazquez, J.J. Meyerle, V.Y. Lee, Phys. Rev. Lett. 46, 253 (1981).

[9] T. Iizuka-Sakano, Y. Toyozawa, in preparation.

[10] S. Koshihara, Y. Tokura, T. Mitani, G. Saito, T. Koda, Phys. Rev. B 42, 6853 (1990). 